\title{
- NOTES-
}

\section{A NOTE ON SINGULARITIES IN A COSSERAT CONTINUUM*}

\author{
By YECHIEL WEITSMAN (Brown University)
}

\begin{abstract}
This paper is concerned with the singularities that are due to concentrated couples in an infinite linear, elastic, isotropic Cosserat continuum. The solution to the problem of a concentrated couple, acting within an infinite region, may be obtained as a limiting case of the solution to the problem of body moments acting within a finite portion of the infinite medium. Alternatively, the solution to the problem of the concentrated couple can be constructed from the solution to the case of a concentrated force acting within an infinite body, by combining two double-forces with moments to form a center of rotation.

In this paper it is shown that in a Cosserat continuum the two above mentioned singular solutions to the case of a concentrated couple, acting within an infinite body, are not the same. By means of a specific linear combination of these two singular solutions it is possible to reconstruct the classical center of rotation, which is accompanied by an additional micro-rotation field. It is shown that there exists a limiting case in which the macro-displacements are eliminated altogether, resulting in a singular field of micro-rotations alone.
\end{abstract}

1. The equations of a linear, elastic, isotropic Cosserat continuum. In a Cosserat continuum [1] ${ }^{* *}$, deformations are characterized by two kinematical variables: the displacement $u_{i}$ and the independent, rigid, anti-symmetric micro-rotation $\psi_{[i i]}$. The quantities $\psi_{[i i]}$ describe a rigid rotation of some material "superstructural" property (e.g. the Cosserat triad or, alternatively, a "micro-structure").

Following Mindlin's formulation [2] of the linear, elastic case we define

$$
\begin{aligned}
\epsilon_{i j} & =\frac{1}{2}\left(u_{i, i}+u_{i, i}\right), \\
\gamma_{[i i]} & =\frac{1}{2}\left(u_{i, i}-u_{i, i}\right)-\psi_{(i i)}, \\
\kappa_{i[i k]} & =\psi_{(i k\}, i} .
\end{aligned}
$$

Then, for an isotropic, centrosymmetric medium the constituitive relations are

$$
\begin{aligned}
\tau_{i j} & =\lambda \epsilon_{k k} \delta_{i j}+2 \mu \epsilon_{i j}, \\
\sigma_{[i i]} & =2 \beta \gamma_{[i i]}, \\
\mu_{i\lfloor i k]} & =\alpha_{1}\left(\kappa_{l[l i]} \delta_{i k}+\kappa_{l[k l]} \delta_{i j}\right)+2 \alpha_{2} \kappa_{i[i k]}+\alpha_{3}\left(\kappa_{k[i j]}+\kappa_{i[k i]}\right),
\end{aligned}
$$

where $\tau_{i j}$ is the classical "Cauchy" stress, $\sigma_{[i i]}$ is the anti-symmetric part of Mindlin's relative stress, and $\mu_{i(i k]}$ is the Cosserat's couple-stress. The quantities $\alpha_{i}$ and $\beta$ are

*Received February 21, 1966; revised manuscript received May 23, 1966.

**Numbers in square brackets indicate the reference listed at the end of this paper. 
some material constants. The dimension of $\alpha_{i}$ differs from the dimension of the Lame constants $\lambda, \mu$ by the square of length.

The equations of equilibrium read:

$$
\begin{aligned}
\left(\tau_{i j}+\sigma_{[i i]}\right)_{, i}+f_{i} & =0, \\
\mu_{i[i k], i}+\sigma_{[i k]}+\phi_{[j k]} & =0 .
\end{aligned}
$$

In (3) $f_{i}$ and $\phi_{[i k]}$ denote body force and body couple, respectively.

The boundary conditions, at a boundary with outward normal $n_{i}$, are

$$
\begin{aligned}
t_{i} & =n_{i}\left(\tau_{i j}+\sigma_{[i i]}\right), \\
T_{[i k]} & =n_{i} \mu_{i[j k]} .
\end{aligned}
$$

Substituting (1) in (2), and then in (3) we obtain the kinematical equations of motion

$$
\begin{gathered}
(\lambda+\mu-\beta) u_{i, j i}+(\mu+\beta) u_{i, j i}-2 \beta \psi_{[i i], i}+f_{i}=0 \\
\left(\alpha_{1}+\alpha_{3}\right)\left(\psi_{[k i], k i}+\psi_{[i k\}, k i}\right)+2 \alpha_{2} \psi_{[i j], k k}-2 \beta \psi_{[i j]}+\beta\left(u_{i, i}-u_{i, j}\right)+\phi_{[i j]}=0
\end{gathered}
$$

Employing the "direct" notation, Eqs. (5) read

$$
(\lambda+\mu-\beta) \nabla \nabla \cdot \mathbf{u}+(\mu+\beta) \nabla^{2} \mathbf{u}-2 \beta \nabla \cdot \mathbf{\psi}^{\Lambda}+\mathbf{f}=0,
$$$$
\left(\alpha_{1}+\alpha_{3}\right)\left(\nabla \cdot \psi^{A} \nabla+\nabla \psi^{A} \cdot \nabla\right)+2 \alpha_{2} \nabla^{2} \psi^{A}-2 \beta \psi^{A}+\beta(\nabla \mathbf{u}-\mathbf{u} \nabla)-\frac{1}{2} \mathbf{I} \times \mathbf{c}=0 .
$$

In (6) the quantities $\mathbf{u}$ and $\mathbf{f}$ are vectors with components $u_{i}$ and $f_{i}$ and $\boldsymbol{\psi}^{\mathcal{A}}, \phi^{\boldsymbol{A}}$ and I are dyadics with components $\psi_{[i i]}, \phi_{[i i]}$ and $\delta_{i j}$. The quantity $\boldsymbol{\phi}^{\boldsymbol{\Lambda}}$ has been written in terms of a body couple vector $\mathbf{c}$,

$$
\phi^{A}=-\frac{1}{2} \mathrm{I} \times \mathbf{c} .
$$

Mindlin has shown [2], that a complete solution of (6), or (5), can be expressed as

$$
\begin{gathered}
\mathbf{u}=\nabla \times \mathbf{K}+\left(1-l_{3}^{2} \nabla^{2}\right)\left(\mathbf{B}-l_{1}^{2} \nabla \nabla \cdot \mathbf{B}\right)-\frac{1}{2}\left(k_{1}-l_{3}^{2} \nabla^{2}\right) \nabla\left[\mathbf{r} \cdot\left(1-l_{1}^{2} \nabla^{2}\right) \mathbf{B}+B_{0}\right], \\
\boldsymbol{\psi}^{\mathbf{A}}=-\frac{1}{4} \mathbf{I} \times\left[\nabla^{2} \nabla\left(\mathbf{r} \cdot \mathbf{K}+K_{0}\right)+2 \nabla \times \mathbf{B}\right]
\end{gathered}
$$

where $\mathbf{B}, B_{0}, \mathbf{K}$, and $K_{0}$ are stress-functions of the Boussinesq-Papkovitch type. These functions satisfy the following relations:

$$
\begin{gathered}
\mu\left(1-l_{1}^{2} \nabla^{2}\right) \nabla^{2} \mathbf{B}=-\mathbf{f}-\frac{\mu+\beta}{2 \beta} \nabla \times \mathbf{c}, \\
\mu \nabla^{2} B_{0}=\mathbf{r} \cdot\left[\mathbf{f}+\frac{\mu+\beta}{2 \beta} \nabla \times \mathbf{c}\right], \\
2 \beta \nabla^{2} \mathrm{~K}=\mathbf{c}, \\
2 \beta\left(1-l_{2}^{2} \nabla^{2}\right) \nabla^{2} K_{0}=4 l_{2}^{2} \nabla \cdot \mathbf{c}-\mathbf{r} \cdot\left(1-l_{2}^{2} \nabla^{2}\right) \mathbf{c}
\end{gathered}
$$

where, in ( 7 ) and (8)

$$
\begin{aligned}
& k_{1}=\frac{\lambda+\mu}{\lambda+2 \mu}, \\
& l_{1}^{2}=\left(2 \alpha_{2}-\alpha_{1}-\alpha_{3}\right) \frac{\mu+\beta}{2 \mu \beta}, \\
& l_{2}^{2}=\frac{\alpha_{2}}{\beta}, \quad l_{3}^{2}=\frac{2 \alpha_{2}-\alpha_{1}-\alpha_{3}}{2 \beta},
\end{aligned}
$$


2. The singular solutions for concentrated force and couple. Mindlin [2] has given the solution to the problems of a concentrated force and a concentrated couple acting within an infinite medium.

For a concentrated force $\mathrm{P}$, acting at the origin of a Cartesian coordinate system $x, y, z$ the stress-functions are

$$
\begin{aligned}
\mathbf{B} & =\frac{\mathbf{P}}{4 \pi \mu} g_{1}, \\
B_{0} & =0, \\
\mathbf{K} & =0, \\
K_{0} & =0 .
\end{aligned}
$$

For a concentrated moment $\mathbf{C}$, acting at the origin the stress functions are

$$
\begin{aligned}
\mathbf{B} & =-\frac{\mu+\beta}{8 \pi \mu \beta} \mathbf{C} \times \nabla g_{1}, \\
B_{0} & =0, \\
\mathbf{K} & =-\frac{\mathbf{C}}{8 \pi \beta r}, \\
K_{0} & =-\frac{l_{2}^{2}}{4 \pi \beta} \mathbf{C} \cdot \nabla g_{2} .
\end{aligned}
$$

In (9) and (10) $g_{i}=\left(1-e^{-r / l_{i}}\right) / r$.

Let $\mathbf{u}^{(1)}$ and $\psi^{A(1)}$ denote the kinematical field due to a concentrated force $P \mathbf{e}_{x}$ acting at the origin, and $\mathfrak{u}^{(2)}, \psi^{A(2)}$ be the kinematical field that is due to a force $P \mathbf{e}_{v}$ acting at the origin.

Employing the fields $\mathbf{u}^{(1)}, \Psi^{A(1)}$ and $\mathfrak{u}^{(2)}, \Psi^{A(2)}$, it is possible to construct a singular solution due to a "center of rotation about the axis of $z$ " [3]. We let the forces $h^{-1} P \mathbf{e}_{\boldsymbol{x}}$ and $-h^{-1} P \mathbf{e}_{\nu}$ act at the origin $(0,0,0)$, and the forces $-h^{-1} P \mathbf{e}_{x}$ and $h^{-1} P \mathbf{e}_{\nu}$ act at $(0, h, 0)$ and $(h, 0,0)$, respectively, as shown in Fig. 1.

Passing to the limit as $h \rightarrow 0$ the resulting kinematical field is given by

$$
\begin{gathered}
\mathfrak{u}^{(R)}=\frac{\partial \mathbf{u}^{(1)}}{\partial y}-\frac{\partial \mathbf{u}^{(2)}}{\partial x} \\
\psi^{A(R)}=\frac{\partial \mathfrak{\psi}^{A(1)}}{\partial y}-\frac{\partial \mathfrak{\psi}^{A(2)}}{\partial x} .
\end{gathered}
$$

Computing the field $\mathbf{u}^{(R)}$ and $\psi^{A(R)}$ we obtain

$$
\begin{aligned}
\mathbf{u}^{(R)} & =-\frac{P}{4 \pi \mu}\left(1-l_{3}^{2} \nabla^{2}\right) \mathrm{e}_{z} \times \nabla g_{1}, \\
\psi^{A(R)} & =\frac{P}{8 \pi \mu} \mathrm{I} \times \nabla \times \mathrm{e}_{2} \times \nabla g_{1} .
\end{aligned}
$$

Expressions (12) may be obtained from (7) if we select 


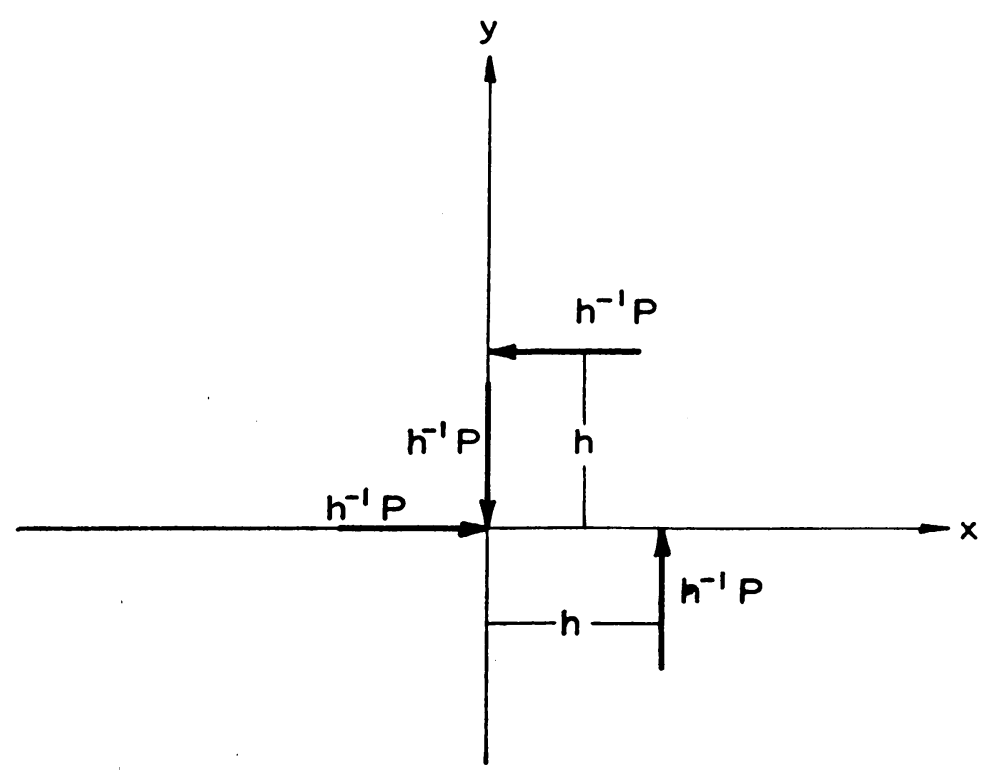

Frg. 1. The system of concentrated forces which yields, in the limit as $h \rightarrow 0$, a "center of rotation about the axis of $z$."

$$
\begin{gathered}
\mathrm{B}=\mathrm{B}^{(R)}=-\frac{P}{4 \pi \mu} \mathrm{e}_{3} \times \nabla g_{1}, \\
B_{0}^{(R)}=\mathrm{K}^{(R)}=K_{0}^{(R)}=0 .
\end{gathered}
$$

For a concentrated moment directed about the $z$ axis, at the origin $\mathbf{C}=C \mathbf{e}_{s}$, Eqs. (10) yield

$$
\begin{aligned}
& \mathrm{B}^{(C)}=-\frac{\mu+\beta}{8 \pi \mu \beta} C \mathrm{e}_{2} \times \nabla g_{1}=\frac{\mu+\beta}{2 \beta} \frac{C}{P} \mathrm{~B}^{(R)}, \\
& B_{0}^{(C)}=0 \\
& \mathrm{~K}^{(C)}=-\frac{C}{8 \pi \beta r} \mathrm{e}_{\mathrm{s}}, \\
& K_{0}^{(C)}=-\frac{l_{2}^{2} C}{4 \pi \beta} \frac{\partial g_{2}}{\partial z} .
\end{aligned}
$$

A comparison between (13) and (14) shows that the singularities due to the two kinds of concentrated couples are not the same. They differ by the stress functions $\mathbf{K}$ and $K_{0}$, given in (14). These functions yield a self-equilibrating kinematical field.

It may be worth noting that in the case of couple-stress theory [4], the singularity due to a concentrated couple and the singularity due to a "center of rotation" are the same.

3. Special cases. (a) Consider the following linear combination of the solutions to a concentrated couple $S^{\left(C^{\prime}\right)}$ and a center of rotation $S^{(R)}$.

$$
S^{(L)}=-\frac{2 P}{C} \frac{\beta}{\mu} S^{(C)}+\frac{\mu+\beta}{\mu} S^{(R)} .
$$


Then

$$
\begin{aligned}
\mathbf{B}^{(L)} & =-\frac{2 P}{C} \frac{\beta}{\mu} \mathbf{B}^{(C)}+\frac{\mu+\beta}{\mu} \mathbf{B}^{(R)}=0, \\
B_{0}^{(L)} & =0 \\
\mathbf{K}^{(L)} & =-\frac{2 P}{C} \frac{\beta}{\mu} \mathbf{K}^{(C)}=\frac{P}{4 \pi \mu} \frac{\mathbf{e}_{z}}{r}, \\
K_{0}^{(L)} & =-\frac{2 P}{C} \frac{\beta}{\mu} K_{0}^{(C)}=\frac{P l_{2}^{2}}{2 \pi \mu} \frac{\partial g_{2}}{\partial z} .
\end{aligned}
$$

The corresponding kinematical field is

$$
\begin{aligned}
\mathfrak{u}^{(L)} & =\frac{P}{4 \pi \mu} \nabla \times \frac{\mathbf{e}_{z}}{r}, \\
\boldsymbol{\psi}^{A(L)} & =\frac{P}{8 \pi \mu} \mathrm{I} \times \nabla \frac{\partial g_{2}}{\partial z} .
\end{aligned}
$$

It is interesting to note that the expression for $\mathbf{u}^{(L)}$ has thus been made to agree with the classical result for a center of rotation about the axis of $z$. It does not depend on the "micro-parameters" of the Cosserat medium.

(b) Consider the limit of the solution $S^{(C)}$, for a concentrated moment about the $z$ axis, as the ratio $\mu / \beta \rightarrow \infty$.

In this case the characteristic lengths $l_{1}^{2} \rightarrow l_{3}^{2}$ and

$$
\left(1-l_{3}^{2} \nabla^{2}\right) g_{1} \rightarrow 1 / r \text {. }
$$

The kinematical fields become

$$
\begin{gathered}
\mathbf{u}^{(C)} \rightarrow 0, \\
\boldsymbol{t}^{A(C)}=-\frac{C}{16 \pi \beta} \mathbf{I} \times\left[\frac{e^{-r / l_{1}}}{r l_{1}^{2}} \mathbf{e}_{z}+\nabla\left(\frac{\partial g_{1}}{\partial z}-\frac{\partial g_{2}}{\partial z}\right)\right] .
\end{gathered}
$$

It is seen that for this limiting case the macro-displacements vanish, and the resulting singular field contains micro-rotations alone.

Acknowledgments. The writer wishes to express his indebtedness to Professor R. D. Mindlin for a most valuable discussion.

This investigation was supported by the Office of Naval Research, Contract Nonr(G)00040-65, Task NRO64-488, and by the Ordnance Research Laboratory at The Pennsylvania State University.

\section{REFERENCES}

1. E. and F. Cosserat, Théorie des corps deformables, Hermann, Paris, 1909

2. R. D. Mindlin, Stress functions for a Cosserat continuum, Int. J. Solids Structures, 1, 265-271 (1965)

3. A. E. H. Love, A treatise on the mathemalical theory of elasticity, Dover, Fourth Ed. (Especially Sec. 132)

4. R. D. Mindlin and H. F. Tiersten, Effects of couple-stresses in linear elasticity, Arch. Rational Mech. Anal., 11, 415-448 (1962) 